\title{
BLACK HOLES
}

\section{Feeling the drag}

Astrophys. J. Lett. (in the press); preprint at https://arxiv.org/abs/2003.11770

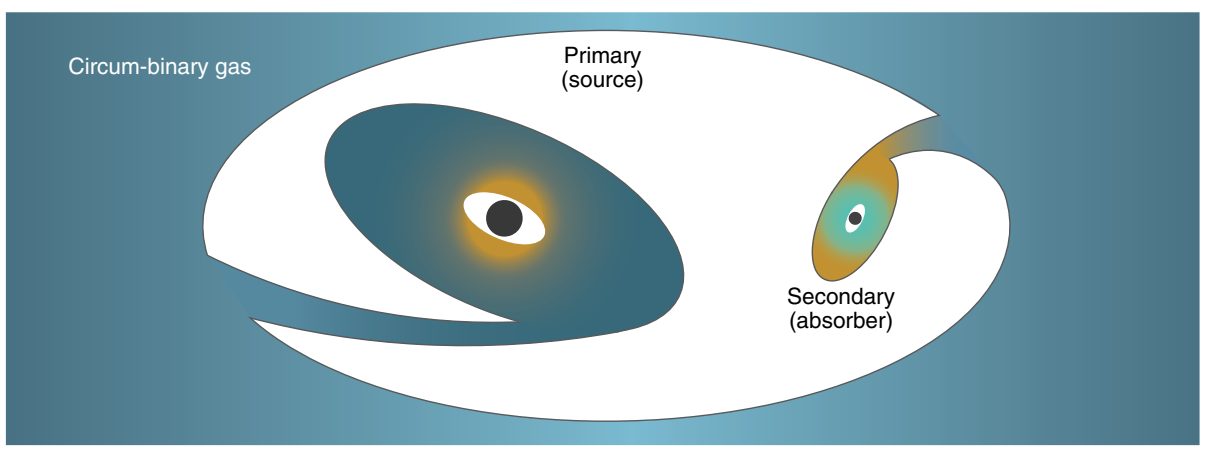

Credit: AAS

Within a hierarchically evolving Universe, binary black holes (BBHs) are expected to form through galaxy mergers. How these $\mathrm{BBH}$ merge is, however, still under debate. Xian Chen and collaborators show that Poynting-Robertson (PR) drag, a radiative process whose effects are observed in the interaction between the Sun and dust in our Solar System, is applicable to BBHs and can play an important role in shrinking their orbits sufficiently for them to merge.

The PR drag in the case of a $\mathrm{BBH}$ is a force exerted on the accretion disk around the secondary black hole, which is being irradiated by the accretion disk of the primary (pictured). As the secondary's accretion disk absorbs the emitted radiation, due to its orbital motion it emits more photons in its direction of travel. As a result of momentum conservation, the secondary black hole and its accretion disk then recoil in the opposite direction. The timescale of the secondary's deceleration depends on the mass ratio of the $\mathrm{BBH}$, the primary's accretion rate and the inclination between the two accretion disks.

Going a step further, Chen et al. show that in the case of an extreme-mass-ratio $\mathrm{BBH}$ (a stellar black hole around a $10^{6}-10^{7}$ $M_{\odot}$ black hole or an intermediate-mass black hole around a $10^{8}-10^{10} M_{\odot}$ black hole), deceleration due to the $\mathrm{PR}$ drag can be faster than that for other processes such as dynamical friction. As the PR-drag timescale is smaller than the typical duty cycle of an active galaxy, it is efficient in bringing $\mathrm{BBHs}$ to coalescence. This work paves the way to implementing this additional, potentially important, effect in future hydrodynamic and cosmological simulations.

\section{Marios Karouzos}

Published online: 15 April 2020

https://doi.org/10.1038/s41550-020-1091-6 